\title{
Native Americans in California surveyed on diets, nutrition needs
}

\author{
Joanne lkeda $\square$ Sharon Dugan $\square$ Nancy Feldman $\square$ Rita Mitchell
}

\begin{abstract}
Investigation of the diets of Native Americans in California's Yosemite-Mariposa region identified eating habits that contributed to their well-being as well as habits that were potentially detrimental. The main problem: economics. With little money and no access to major grocery chains, many families cannot buy the kind of food that supports an adequate diet; others are unaware of government programs that can help them.
\end{abstract}

In California's Native American population, health problems related to poor nutrition are common. Heart disease, the leading cause of death, accounted for $27.4 \%$ of all deaths in 1985-1987; cancer accounted for $14.6 \%$. Diabetes is also widespread; in 1987, the age-adjusted "diabetes mellitus" mortality rate for the Indian Health Service (IHS) in California was 20.8 per 100,000 people, compared with the overall U.S. rate of 9 per 100,000 .

The California Department of Health Services is identifying community and health care objectives, including stepping up the nutrition education of Native Americans to combat the risks of heart disease, cancer and diabetes.

In 1989, the Tuolumne Rural Indian Health Center and the University of California's Cooperative Extension studied the diets of Native Americans served by the Mariposa Indian Health Center to develop a culturally relevant nutrition education and counseling program.

\section{Background}

American Indians have inhabited the Yosemite Valley and the surrounding region for more than 4,000 years. They lived by gathering, hunting and fishing. During the 19th century violent and tragic encounters with settlers decimated their population. Today, some 500 Native Americans live in the Yosemite-Mariposa region, descendants of the Miwok-speaking people.

Nutritionists from UC Berkeley and the Mariposa Indian Health Center staff started out with Ann Hertzler's classification of cultural food habits by content and context. Hertzler, a nutritionist at Virginia Polytechnic Institute and State University, outlined broad categories which need investigation when studying a group's food habits [see 1982 Journal of the American Dietetic Association (80:421-25)]. Based on this, the authors developed 51 questions regarding meal patterns, typical foods consumed, cultural foods, food acquisition, food resources, food preparation, nutrition during pregnancy and special dietary concerns. Furthermore, respondents were re quired to account for everything eaten within a 24-hour period, and to answer questions about their family composition, ethnic background, monthly income, monthly expenses for food and enrollment in government food and nutrition programs.

Indian community health representatives were trained to ask the questions and to recruit 51 Native Americans to participate in the study. The sample represents about $10 \%$ of today's population. Interviews were conducted in the homes of the participants; the community health representatives recorded responses in writing.

The respondents, 45 females and 6 males, ranged in age from 22 to 88 ; the average household consisted of three members. All lived in Mariposa County, the location of Yosemite National Park.

\section{Findings}

Retired UCLA Nutrition Professor Derrick Jelliffe suggested a crosscultural model of nutrition education that categorizes healthy eating habits that should be supported and encouraged, unhealthy habits that should be discouraged and those that can be improved by, for example, increasing amounts consumed. This study followed this method of categorization.

Healthy eating habits were apparent. For example, $80 \%$ reported that family members ate at least one meal together each day. This contrasts with a trend permeating American culture where fewer meals are eaten together by families. $\mathrm{Nu}-$ trition educators have long pointed out that family mealtimes promote good eating habits in the young and that children who eat regular meals are likely to consume better diets than children who do not.

Pregnancy and babies. The nutritional needs of pregnancy were met by the majority of women responding. Of those who had been pregnant at least once, $63 \%$ (24 women) reported eating differently during pregnancy. Asked how they ate differently, $75 \%$ said they ate more fruits and vegetables, $71 \%$ consumed more milk and milk products, $50 \%$ ate more greens and grains/cereals and $29 \%$ ate more animal protein. Only three made possibly detrimental dietary changes; two had increased "sweets," and one drank more soft drinks.

Breastfeeding was practiced by 11 of the 16 women with children under 5 years of age, another positive finding. Historically, lower socioeconomic groups in the U.S. have chosen to bottle-feed their infants. Physicians and other health professionals encourage breastfeeding because of its immunological, emotional and nutritional advantages. Another practice that should be encouraged is delaying the in troduction of solid foods. Seventy-seven percent of the women in the study with children under 5 waited until at least 4 months of age to introduce solid foods.

The Women, Infants, and Children Supplemental Food Program (WIC) apparently influenced four of the seven women who participated in the program in the way they fed their infants. Ten other women, eligible to participate in WIC, did not enroll. Increased participation in WIC by eligible women needs encouragement.

Drinking milk with meals was another beneficial eating habit reported; $61 \%$ of participants in the study and $85 \%$ of the children in their families reportedly drank milk with meals. Analysis of the subjects' 24-hour food recalls shows a median calcium intake of $518 \mathrm{mg}$, which is $56 \%$ of the recommended dietary allowance (RDA) the amount recommended for optimum health. The nutrient density of the diet for calcium was adequate at $418 \mathrm{mg}$ per 1,000 calories.

Soft drink consumption, which is rising in the U.S., was evident in the survey. Thirty-three percent said they drank soda with meals; $26 \%$ reported that their children drank soft drinks at mealtime. For 


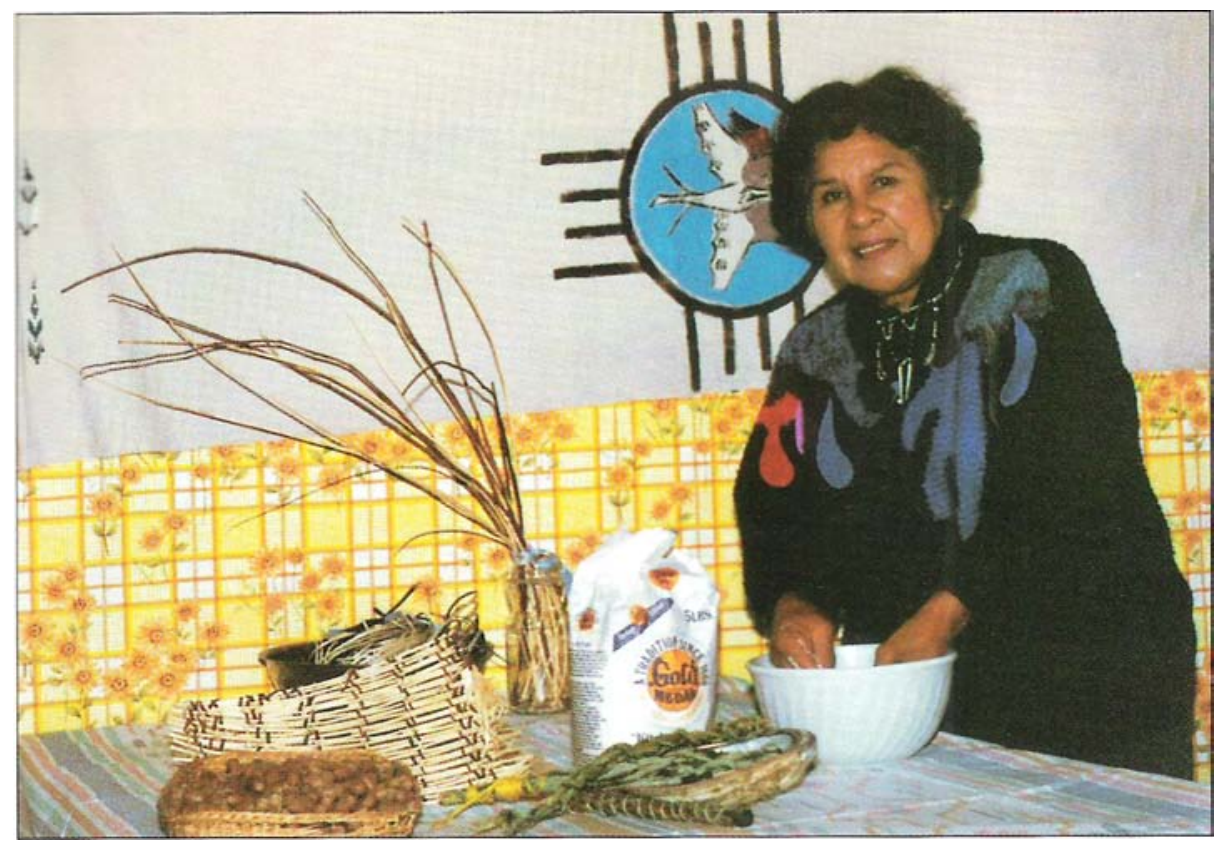

Helen Coats, community health representative for Mariposa Indian Health Center, demonstrates how to make fry bread with less fat and salt and the added ingredient of nonfat dry milk. The new recipe was developed in response to the authors' findings.

those who cannot tolerate drinking milk, nutrition educators suggest the consumption of other calcium-rich foods.

Stretching dollars. These Native Americans used several strategies to stretch their food dollars. One hundred percent reported buying "on sale" food items regularly; $82 \%$ often used a list when shopping, and $77 \%$ frequently used "cents-off" discount coupons. Still, 81\% wanted to know about other ways they could save money when grocery shopping.

Some families augmented their food supply in traditional ways: $47 \%$ gathered wild berries, nuts, mushrooms and other plants; $67 \%$ said their grandparents and parents traditionally used wild plants as foods, and $81 \%$ said this knowledge had been passed on to them by their elders. Twenty-two percent said they had a garden that provided them with fruits and vegetables. With respect to fishing and hunting, $26 \%$ reported someone in their family fished, and 14\% said.someone hunted deer, rabbit and squirrel.

These strategies were necessary as $50 \%$ of the respondents lived below the federal poverty level (an income of $\$ 13,400$ for a family of four) and $70 \%$ had incomes within $125 \%$ of the poverty level $\$ \$ 16,750$ for a family of four). The average family of three spent $\$ 308.20$ for food each month, compared with the U.S. Department of Agriculture "low-cost" food plan for a family of three of $\$ 341.04$. These families live in a rural area where access to lowcost foods is limited. Even augmenting food supplies by hunting, fishing, gardening and gathering was not enough. Thirty percent said there were times when there was no food or money and family members went hungry. Other evidence of lim- ited food resources: $39 \%$ reported eating one or two meals a day. Many (65\%) said they would eat differently if they had more money for food; more than half indicated that they would buy more fresh produce.

\section{Discussion}

Promoting participation in federally sponsored food and nutrition programs is one way of increasing the food resources of these families. Even though $70 \%$ of the families were eligible for food stamps, only $33 \%$ took part in the program, and, as gible respondents were enrolled in WIC. This study did not examine the possible barriers to participation in these programs; it would certainly be pertinent to identify why people are not taking advantage of them.

Calorie intake. Computer analysis of the participants' 24-hour food recalls revealed a median calorie intake of 1,238 calories, low compared to the U.S. median of about 1,400 calories (with a range of 900 to 2,000). Because calorie intake was low, median RDA's were low, and median nutrient intake for 7 vitamins and minerals was less than two-thirds of the RDA.

Although these data appear to confirm this population's limited resources for obtaining food, they also raise the question of the accuracy of the recalls. Although the interviewers were trained to take and record food recalls, they may have felt uncomfortable asking probing questions. When the low calorie intakes for the 24hour food recalls were discussed with them, they responded that they felt people were eating more snacks in the evening than they reported. Because the reliability of these data is in question, there is limited reporting of the results. mentioned previously, only 7 out of 17 eli-
Computer analysis of macronutrient intake revealed that the median percentage of calories from fat ingested was $36 \%$, compared with the $30 \%$ recommended in the recently published U.S. Dietary Guidelines for Americans. Median sodium intake, as calculated from computer analysis of the 24-hour food recalls, was 2,308 $\mathrm{mg}$, an acceptable amount when compared with the limit of $2,400 \mathrm{mg}$ recommended by the Food and Nutrition Board of the National Academy of Sciences. However, true sodium intake may be higher, as $70 \%$ of the respondents said they added salt in food preparation, and $73 \%$ indicated that they or someone else in the household sprinkled salt on food at the table. Furthermore, $61 \%$ reported they were overweight. No data were collected to confirm this, but a combination of high sodium intake and obesity can increase the risk of hypertension.

One habit that can contribute to overweight is snacking while watching television. Sixty-nine percent of the participants snacked in the evening; of those who snacked, $85 \%$ did so while watching TV. When asked about snack foods eaten every day or almost every day, the most frequently mentioned snack was fruit, followed by soft drinks, potato or corn chips, cookies and cakes, and ice cream. Snacking on fruit should, of course, be encouraged, but the other snack choices are of little nutritive value and could be replaced with more nutritious choices.

Traditional foods. Asked about foods they think of as Native American foods, study participants listed beans, rice, tortillas, fry bread, enchiladas, tacos, Indian tacos and venison in descending order. Foods associated with pow-wows included acorns, venison, Indian tacos, salmon, pine nuts, beans and fry bread in descending order. Acorns, pine nuts, and venison were Miwok staple foods before the early 1900s.

Many Miwok recalled foods their grandparents ate that they do not eat: insects such as pine tree worms, Monarch butterfly larvae and grasshoppers; animals like squirrel, Mono Lake shrimp, quail, deer, rabbit, bear and hedge hog; and plant foods such as acorn mush, pine nuts, wild vegetables and berries. Some of these foods, particularly the insects, are not considered food by the dominant culture. This may have influenced these Native Americans to abandon them as food sources.

The Miwok have limited access to wild game and plant foods because of hunting restrictions and private ownership of land. There is no Indian reservation or rancheria in this area.

Education needs. Along with wanting to learn more about saving money when shopping for food, respondents were also interested in learning to prepare 
healthy foods (75\%), and learning about healthy foods for their families (72\%). Five respondents were on diabetic diets, and three were on weight-loss regimens. Half of those on a special diet indicated that they needed help in adhering to the diet.

When asked, "How would you like to learn more about nutrition?", $69 \%$ asked for newsletters, and $67 \%$ preferred videotape (47\% had a VCR). (Respondents could check more than one method.) Twenty-six percent chose a visit from a health representative; $6 \%$ selected a workshop in the principal author's home with family and friends.

Based on these results, the local Cooperative Extension home economist, working with the Mariposa Indian Health Center staff, produced a videotape entitled, "Native American Recipes Made Healthier - But Still Tasty." The videotape features Miwok homemakers demonstrating traditional ways of preparing deer meat gravy, fry bread, honey berries and vegetables. The homemakers show ways to modify recipes so that they are lower in fat, sodium and sugar, and richer in vitamins and minerals. The videotape has been well received in the Mariposa Native American community. Community leaders have commented that they are pleased with the development of an educational tool that addresses the specific needs and interests of Native American families.

\section{Summary}

In summary, the Native Americans living in California's Mariposa-Yosemite region report a number of positive eating habits that contribute to their well-being. A few nutritionally detrimental practices may need altering. Reinforcing positive food practices and helping Native Americans adopt alternatives to poor eating habits can reduce health risks. Native Americans living in the Mariposa-Yosemite area have limited opportunities to obtain the foods they need and appear to need information on obtaining help from federally sponsored food and nutrition programs. Finally, a videotape that addresses the expressed nutrition needs and interests of the community has been developed for use in the Mariposa American Indian community.

J. Ikeda is Nutrition Education Specialist, Department of Nutritional Sciences, UC Berkeley; S. Dugan is retired Registered Nurse with Tuolumne Rural Indian Health Project; $N$. Feldman is Cooperative Extension Home Economist, Stanislaus and Tuolumne counties, and R. Mitchell is Nutrition Research Associate, Department of Nutritional Sciences, UC Berkeley, and Tuolumne Rural Indian Health Project.

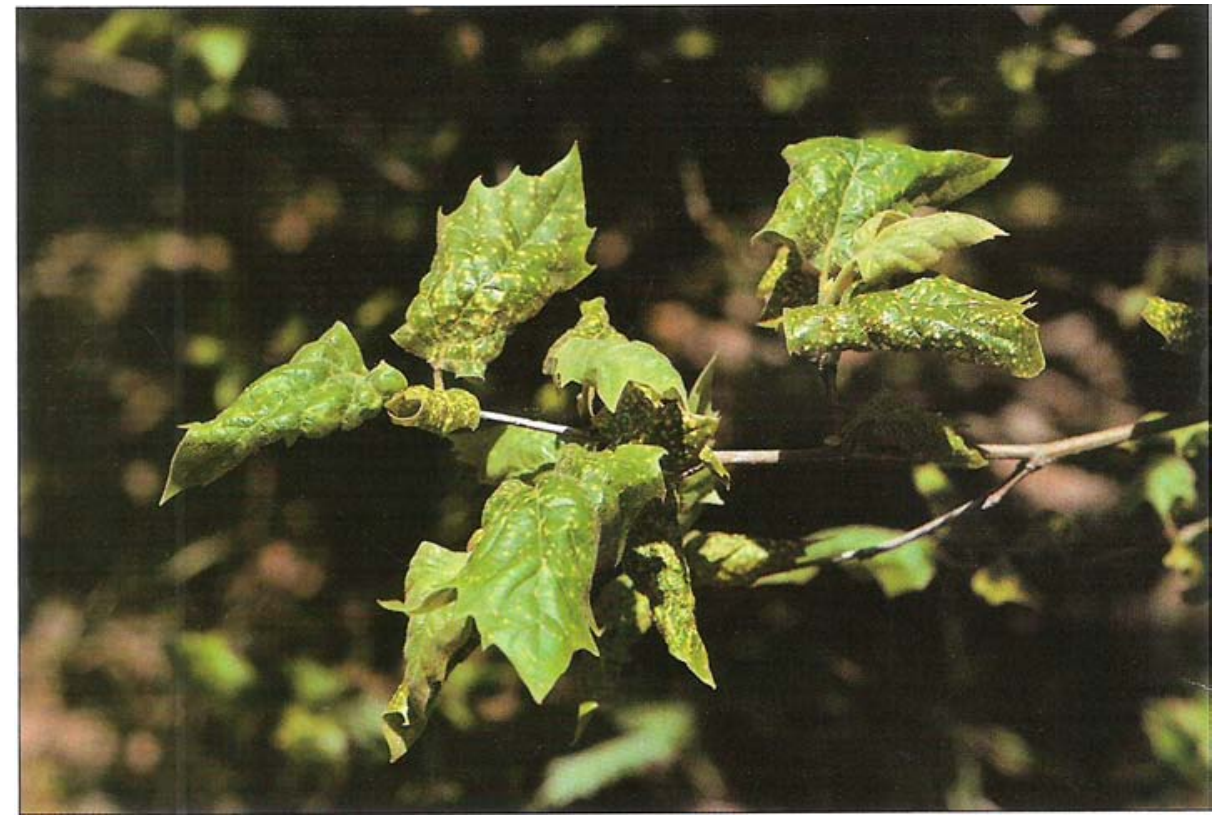

Sycamore scale infestation on untreated trees is seen here.

\section{Sycamore scale treatments most effective at bud break}

\section{Pavel Svihra}

\section{Bud break, not a calendar date, is the best time to protect sycamore trees against the sycamore scale, Stomacoccus platani, with insecti- cidal sprays. It is at bud break that the highest density of crawlers ap. pears on twigs.}

Both native and introduced sycamores (Platanus spp.) can be infested by the sycamore scale, Stomacoccus platani Ferris. Considered the most important insect pest of landscape sycamore trees in California, this native scale also occurs in Nevada.

The most visible injury is the spotting and distortion of leaves caused by scale insects' feeding and the premature loss of portions of such foliage. Less known is the effect of bark feeding by a substantial scale population.

Earlier researchers outlined the seasonal history of the sycamore scale as follows: Except for exposed scales, the insects overwinter on woody tree parts in bark fissures and beneath bark flakes and plates. Eggs are laid there late in winter and crawlers move to new leaves as they appear. After they finish feeding, mature individuals leave their substrate, mate, and the females deposit eggs in masses, mostly on woody parts of trees, to begin the next generation. This cycle is repeated several times during the growing season.

The standard measure for sycamore scale control, developed in Southern California, has been the application of horticultural oil during January, the dormant season. The application reportedly coincides well with the occurrence of the first or second instars, when they are particularly susceptible to oil. Also, application during the dormant period ensures maximum coverage of trees owing to the absence of leaves at that time.

In Northern California, however, pest control applicators have expressed disappointment with the degree of scale control achieved with dormant season sprays of oil. Complaints usually focused on the high levels of leaf loss during the growing season, not the extent of leaf spotting or distortions noted.

The timing of sprays to control sycamore scale in Northern California was 